\title{
Multi-physical processes in geomechanics - an introduction to constitutive modelling and coupling aspects
}

Robert Charlier $_{*}^{*}-$ Arnaud Dizier $^{* * * * *}-$ Lyesse Laloui $^{* * *}-$ Frédéric $^{*}$ Collin "

* Université de Liège

Institut de Génie Civil et de Mécanique

Chemin des Chevreuils 1, 4000 Liège

Sart Tilman, Belgique

Robert.Charlier@ulg.ac.be

**F.R.I.A., FRS-FNRS Fonds National de la Recherche Scientifique

*** Ecole polytechnique fédérale de Lausanne, EPFL - Soil mechanics laboratory

**** FRS-FNRS Fonds National de la Recherche Scientifique

ABSTRACT. This paper is the basis for a course dedicated to the geomechanics modelling, taking into account multiphysics couplings. A number of different coupling are discussed, with respectively the fluid flow (saturated and unsaturated) and the thermal transfers in deformable porous media. Eventually some aspects on the numerical modelling with the finite element method are discussed.

RÉSUMÉ. Cet article est la base d'un cours relatif à la modélisation en géomécanique, avec prise en compte des couplages multiphysiques. Divers couplages avec des écoulements de fluides en régime saturé ou non et avec les transferts thermiques sont pris en compte dans des milieux poreux déformables. Enfin, certains aspects spécifiques relatifs à la modélisation aux éléments finis sont discutés.

KEY WORDS: geomechanics, partial saturation, thermo-plasticity, finite elements, multi-physics coupling.

MOTS-CLÉS: géomécanique, saturation partielle, thermoplasticité, méthode des éléments finis, couplages multiphysiques.

Title of the journal. Volume $\mathrm{X}$ - no X/2002, pages 1 to $\mathrm{n}$ 


\section{Introduction}

This paper is intended as the basis for a course on coupled poromechanics processes and their numerical modelling with the finite element method.

A number of constitutive models have been proposed during the last two decades to describe separately the behaviour of soils submitted to suction (unsaturated soil mechanics), to temperature (thermo-mechanics) as well as to chemistry (chemomechanics) loadings. Such solicitations are the main acting forces in the field of environmental geomechanics (Vulliet et al., 2002). For unsaturated soil mechanics, the Barcelona Basic Model (Alonso et al., 1990) was a starting point for a large number of researches. Among others, (Collin et al., 2002) proposed adaptations of the latter model for rock with fine porosity, e.g. chalk. A chemo-plasticity model was proposed by (Hueckel, 1997) for clay. It was later reworked by (Boukpeti et al., 2003) and (Liu et al., 2005). Other contributions were proposed e.g. on rock weathering by (Nova and Parma, 2005). Thermo-plastic models were developed by (Hueckel and Borsetto, 1990), (Laloui, 1993), (Modaressi and Laloui, 1997). All these contributions are complementary, and some interest was put recently on coupling between two of such environmental loadings and mechanics, e.g. thermomechanics of unsaturated soils or chemo-mechanics of unsaturated soils. The question now arrives on how to experiment in the lab for such multi-coupled phenomena and how to build adequate constitutive frameworks.

This paper intends to present a unified approach to point out a general framework for expressing the constitutive modelling of soils in geo-environmental conditions. From the authors' point of view, this should help to analyse and to model different multi-physics phenomena, and to develop new multi-coupling approaches. We will first derive a generic form for all these constitutive models, and then consider the main effects of each of those geo-environmental loadings.

After this introduction, two sections will be devoted to basic phenomena in partially saturated geomaterial mechanics and in geomaterials thermo-mechanics. Then these notions will be generalised and fully formulated in the elastoplastic framework. The last section is devoted to the finite element modelling of coupled multiphysics processes, and will point the specificities and challenges of such modelling tools.

The ALERT Autumn Schools 2001 and 2005 have been devoted to Multiphysics coupling in geomaterials. The ALERT Autumn Schools 2006 has been devoted to Geomechanics for energy production. The comprehensive courses notes have been published (Laloui et al., 2005a), (Gens and Charlier, 2001), (Charlier et al., 2001) and (Charlier et al., 2006). The reader may find much more details then in this paper which party a synthesis from these books. 


\section{Plasticity for coupled processes: Partial saturation}

\subsection{Introduction}

We consider here soils, rocks or concretes partly saturated by two fluids: generally water and air, but also oil and water (in petroleum engineering) or any other fluid couple. The wetting fluid (indices $w$, often water) is in full contact with the solid skeleton, while the non-wetting fluid (indices $n w$, often gas) remains captive in bubbles, or in a continuous phase, depending of the saturation degree, whose boundary are stable thanks to superficial tensions and capillary menisci.

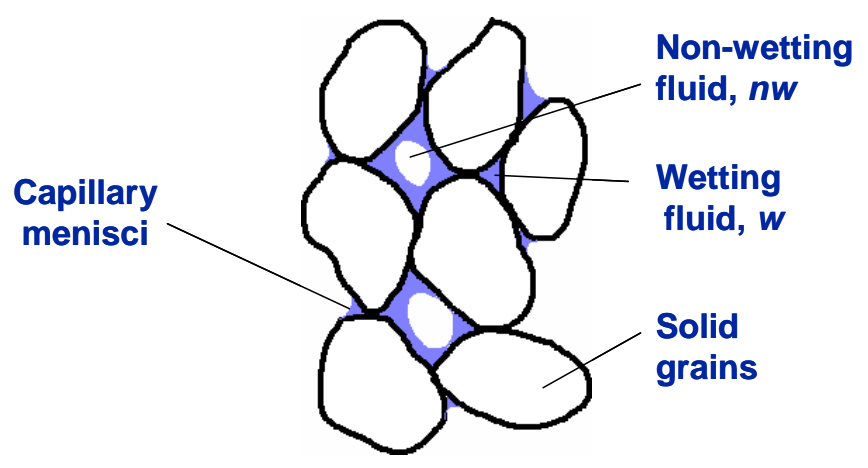

Figure 1. Schematic view of granular geomaterial with two fluids

These superficial tensions are related to the so-called suction $s$ :

$$
s=p_{n w}-p_{w}
$$

Suction is not only due to capillary effects (called matrix suction) but also to osmotic effects and more generally to chemical disequilibria. The superficial tensions are attracting the soil grains and creating an additional inter-granular stress. Any saturation variation induces a variation of suction, of internal stress state and of strain.

Figure 2 (Laloui and Nuth, 2005b) shows a representation of the possible stress paths that the material may experience (respecting the traditional soil mechanics sign convention: compression being positive). In addition to the usual triaxial plane expressed in terms of mean effective stress, $p$ ', and deviatoric stress, $q$, a third axis is added to capture the variations of suction $s$. 


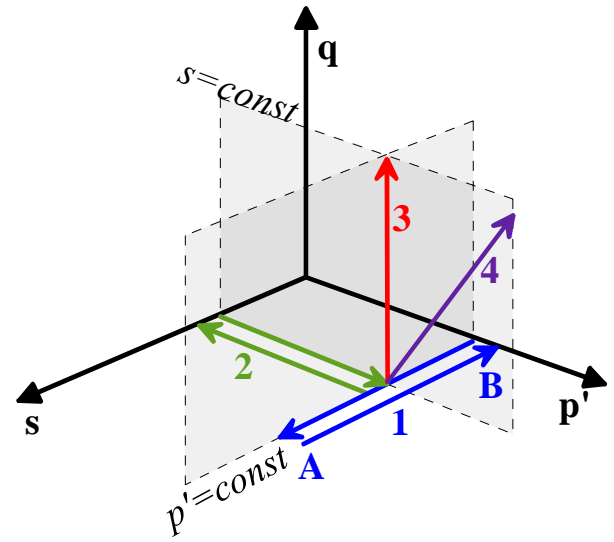

Figure 2. Possible stress paths in $\left(p^{\prime}, q, s\right)$ space

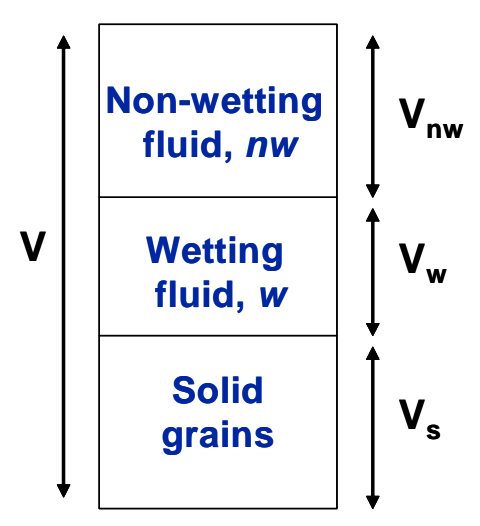

Figure 3. Volume fractions : distribution of solid, wetting and nonwetting fluids in unsaturated soil

The effective stress concept, introduced by Terzaghi (1943), has to be revisited. Terzaghi considered full water saturation and incompressible grains. Biot (1949) pointed out that fluid and matrix compressibility induces some additional coefficients ('the Biot coefficients' as referred by Detournay and Cheng, 1993) and proposed an extended effective stress concept for saturated media. This can not be overcome e.g. for deep rocks. Assuming the volume distribution in the porous medium as illustrated in figure 3, these effective stress concepts have been extended for unsaturated media for a long time e.g. by (Bishops, 1959), who proposed to write the effective stress as:

$$
\sigma_{i j}^{\prime}=\left(\sigma_{i j}-p_{n w} \delta_{i j}\right)+\chi\left(p_{n w}-p_{w}\right) \delta_{i j}
$$

The effective stress parameter $\chi$ takes into account the volume ratio between the phases (degree of saturation $S_{r}$ ) as well as their compressiblities and surface tensions. A simplified version of this Bishop's effective stress was proposed by (Schrefler, 1984):

$$
\sigma_{i j}^{\prime}=\left(\sigma_{i j}-p_{n w} \delta_{i j}\right)+S_{r}\left(p_{n w}-p_{w}\right) \delta_{i j}=\left(\sigma_{i j}-p_{n w} \delta_{i j}\right)+S_{r} s \delta_{i j}
$$

The effective stress concept has been discussed in details by various authors (Coussy, 1995; Nuth et al., 2008).

A wetting process (e.g. a water injection) corresponds to a suction decrease. Following equations [2] or [3], at a constant total stress level, the Bishop's effective stress decreases and the strain is a dilatation or a swelling one in the case of the 
poroelasticity, what is already observed for soils and rock at a given stress conditions (reduced stress level). For other stress conditions (higher stress level), the wetting strains are compressive ones, what is generally called collapse strains. This is often explained by the rupture of the larger pores. To explain the collapse in the framework of Bishop effective stress, this latter should be coupled to a poroplastic constitutive behaviour.

To properly describe the role of the effective stress parameter $\chi$, (Fredlund and Morgenstern, 1977) suggested adopting, instead the Bishop effective stress, two independent stress variables among the total stress, the pore wetting and pore nonwetting stresses. (Alonso et al., 1990) have first proposed an integrated elastoplastic model for taking into account such wetting - collapse behaviour using the net stress (total stress minus the pore non-wetting stress) and the suction. Their model, also called Barcelona Basic Model, is shortly described in the following.

Elastoplasticity is considered, i.e. strain supports an additive decomposition into reversible - elastic and irreversible - plastic parts:

$$
\dot{\varepsilon}_{i j}=\dot{\varepsilon}_{i j}^{e}+\dot{\varepsilon}_{i j}^{p}
$$

Strain variations are induced by either stress variation (denoted by $m$ exponent) or by a variation of the suction (denoted by $s$ exponent). The strain rate writes:

$$
\begin{gathered}
\dot{\varepsilon}_{i j}=\dot{\varepsilon}_{i j}^{m}+\dot{\varepsilon}_{i j}^{s} \\
\dot{\varepsilon}_{i j}^{m}=\dot{\varepsilon}_{i j}^{e, m}+\dot{\varepsilon}_{i j}^{p, m} \\
\dot{\varepsilon}_{i j}^{s}=\dot{\varepsilon}_{i j}^{e, s}+\dot{\varepsilon}_{i j}^{p, s}
\end{gathered}
$$

\subsection{Elasticity}

The elastic strain induced by the coupling variable $s$ rate is a purely volumetric one:

$$
\dot{\varepsilon}_{i j}^{e, s}=K^{s} \dot{s} \delta_{i j}
$$

It may be a non-linear relation, i.e. $K^{s}$ may depend on the stress and coupling variable states. Often, for clayey materials, an oedometer like law is considered, but this has to be adapted to the actual material answer:

$$
\dot{\varepsilon}_{v}^{e, s}=\frac{\kappa_{S}}{1+e_{0}} \frac{\dot{s}}{s}
$$




\subsection{Plasticity}

As shown in figure 4, on an isotropic stress path, elastic as well as plastic strains appear. Generally, for clays, the preconsolidation stress, i.e. the yield stress, and the plastic slope depends on the suction level. This gives the shape of the yield surface (elastic domain) in the $p$ (net stress)-s (suction) space.
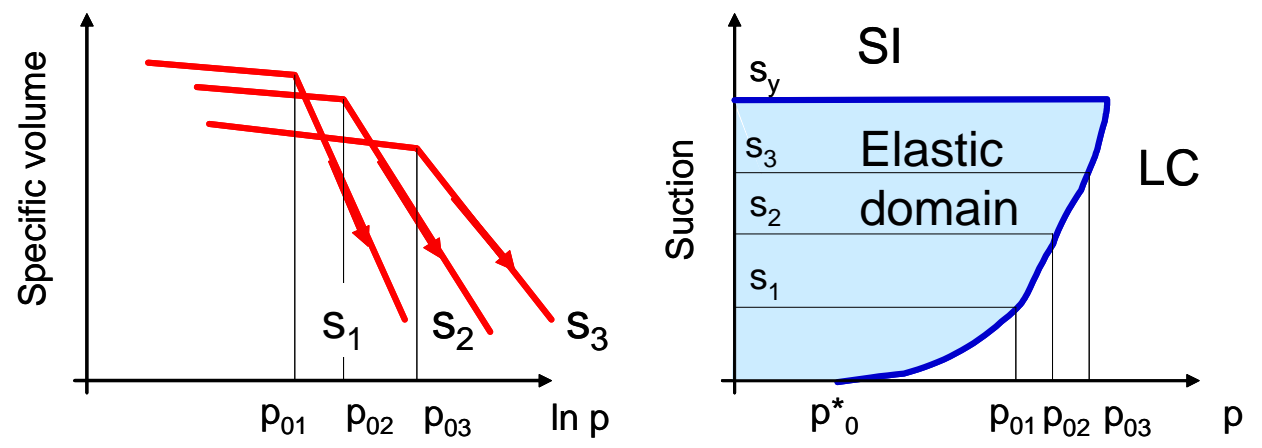

Figure 4. Isotropic mechanical loading at constant suctions: determination of the Loading Collapse (LC) yield curve in the ( $p-s)$ plane.

Generally speaking, the preconsolidation pressure, i.e. the yield threshold for an isotropic loading may be written as:

$$
p_{0}=F_{1}(s) \mathrm{F}_{2}\left(\beta \varepsilon_{v}^{p}\right)
$$

This means that two isotropic hardening mechanisms exist, a suction one and a mechanical / strain one. These processes are not coupled but only combined through a multiplicative function. The mechanical hardening takes the classical form for cohesive soils, as indicated in oedometer tests:

$$
p_{0}=F_{1}(s) \exp \left\{\beta \varepsilon_{v}^{p}\right\}=p_{c 0}(s) \exp \left\{\beta \varepsilon_{v}^{p}\right\}
$$

$p_{c 0}(s)$ is the value of the preconsolidation pressure at suction $s, \beta=\frac{\lambda-\kappa}{1+e}$ is the plastic compressibility modulus. An evolution of the preconsolidation pressure, $p_{c 0}(s)$, is introduced to take into account the suction effect on the yield limit (Laloui et al., 2008):

$$
p_{c 0}(s)=p_{c 0}\left(s_{0}\right)\left\{1+\gamma_{s} \log \left[s / s_{e}\right]\right\}
$$


With $p_{c 0}\left(s_{e}\right)$ the value of the preconsolidation stress at the suction air entry value $s_{e} \cdot \gamma_{s}$ is a material parameter.

The flow rule is associated:

$$
\begin{gathered}
f_{T i}\left(p, s, \varepsilon_{v}^{p}\right)=g_{T i}\left(p, s, \varepsilon_{v}^{p}\right) \\
\dot{\varepsilon}_{i i}^{p}=\lambda_{i} \frac{\partial g_{s i}}{\partial \sigma_{i i}^{\prime}}=\frac{\lambda_{i}}{3}
\end{gathered}
$$

The plastic multiplier, $\lambda_{i}$, is determined using the consistency equation.

With regards to friction angle dependency on suction, Nuth and Laloui (2008) showed the possible evolution of this parameter as well as the apparent cohesion depending on the effective stress representation.

\subsection{Fluid transfers}

Two or more fluids flow in the pore space. It is generally considered that each one follows a Darcy like equation, generalised by Richards for the water - air mixture. The relative fluid velocity $q_{w}$ writes:

$$
\underline{q}^{(i)}=-\frac{\underline{k}^{(i)}}{\mu^{(i)}}\left[\underline{\operatorname{grad}} p^{(i)}+\rho^{(i)} \underline{g}\right]
$$

where the upperscript (i) indicates the fluid (wetting versus non-wetting, oil versus gas, oil versus water...), $\underline{q}$ is the fluid Darcy velocity, $p$ the pressure, $\mu$ the fluid viscosity, $\rho$ the fluid density and $\underline{g}$ the gravity vector. The intrinsic permeability tensor is noted $\underline{k}^{(i)}$. The main change in the Darcy law for partial saturation is a dependency of the permeability $\underline{\underline{k}}^{(i)}$ on the saturation degree $S_{r}^{(i)}$ :

$$
\underline{\underline{k}}^{(i)}=\underline{\underline{k}}^{(i)}\left(S_{r}^{(i)}\right)
$$

Fluid mass balance equation includes classically fluid flows contribution and a sink/source term. For a unit mixture volume, fluid mass balance equation reads:

$$
\frac{\partial\left(S_{r}^{(i)} \rho^{(i)} n\right)}{\partial t}+\operatorname{div}\left(\underline{j}^{(i)}\right)=f^{(i)}
$$

where $j$ is the fluid mass flow $(\underline{j}=\rho \underline{q})$ and $f$ is a mass rate of production/injection of fluid. The fluid storage $S_{r}^{(i)} \rho^{(i)} n$ depends on the saturation degree, on the fluid density and on the change of the pore volume, i.e. on the strain. 
The porosity evolution is a term coupling the mechanics and the flow. If the fluid density changes, it has to be added in the storage evolution. The saturation degree depends itself on the suction:

$$
S_{r}^{(i)}=S_{r}^{(i)}(s)
$$

This function is highly non-linear. A typical curve is given in figure 5 . Moreover, wetting and drying paths give different curves. In soil mechanics, a number of expressions have been given for eq. [15] by various authors.

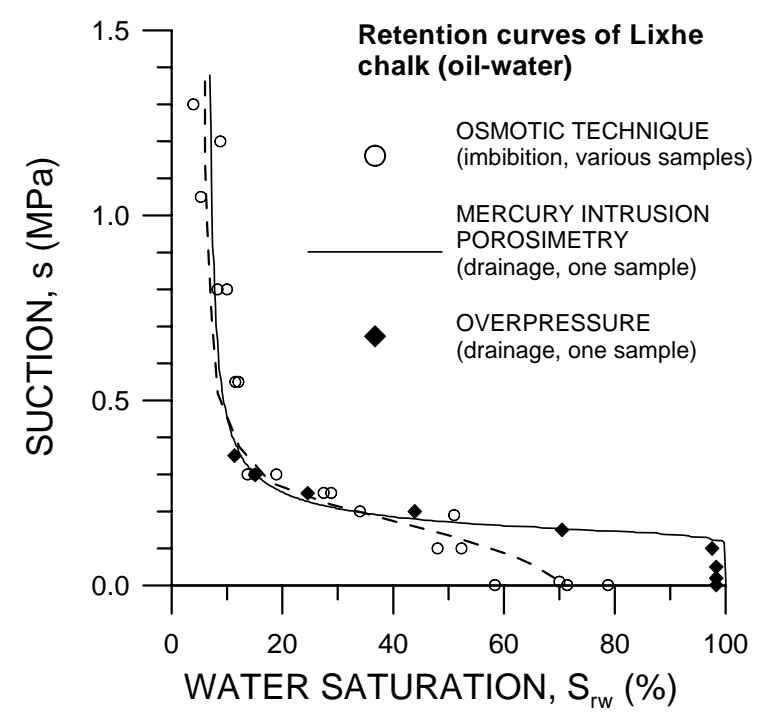

Figure 5. Oil-water retention of Lixhe Chalk (Priol et al. 2004)

\section{Plasticity for coupled processes: Thermoplasticity}

\subsection{Introduction}

The development of the thermoplasticity follows intentionally a framework as similar as possible to the unsaturated elastoplasticity of §2. Effectively, the two formalisms are very similar, for the transfer problem and for the constitutive law itself (Collin et al., 2005). Most published research on thermo-mechanics of geomaterials concerns water saturated clay material. What follows is partly issued from a synthesis done by (Laloui et al., 2005c).

When saturated geomaterials are heated, all of the solid and fluid constituents dilate. In the case of cohesive soils (silt and clay), this dilation produces a decrease 
in the strength of the adsorbed layers and a modification of the distance between the clay particles (Fleureau, 1979). In normally consolidated conditions (NC), the cohesive soils contract when they are heated and a significant part of this deformation is irreversible upon cooling. This thermal contraction is an unusual behaviour for any material. It is related to the microstructure and to the equilibrium of absorbed water layers. Figure 6 illustrates such results: the response to a thermal heating-cooling cycle at constant isotropic stress of a sample of saturated, drained clay (Laloui and Cekerevac, 2008).

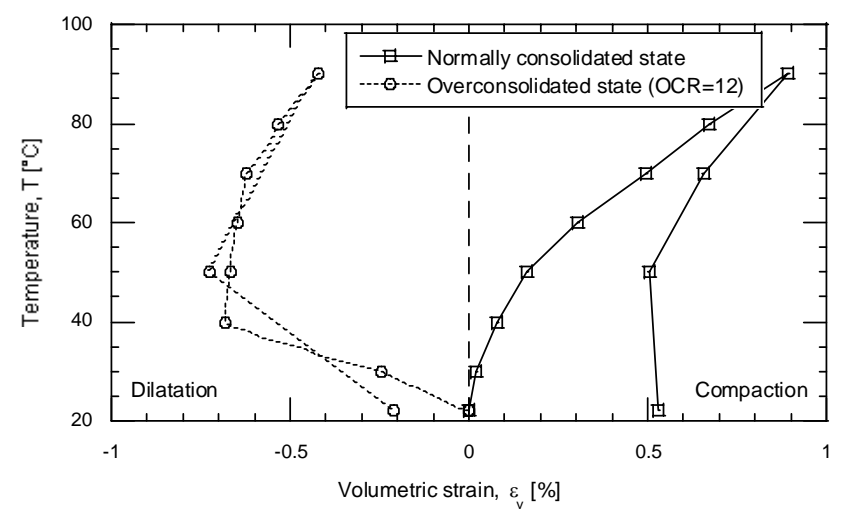

Figure 6. Typical thermal behaviour of fine soils during a thermal heating-cooling cycle - Kaolin clay (Laloui \& Cekerevac, 2008)

As introduced in the section 2.3, preconsolidation pressure, $p_{0}$, is considered here as the stress yield limit which separates "elastic" pre-yield from "plastic" post-yield behaviour in isotropic or oedometer conditions. Several results from the literature show a decreasing of preconsolidation pressure with increasing temperature, figure 7 (Laloui and Cekerevac, 2003).

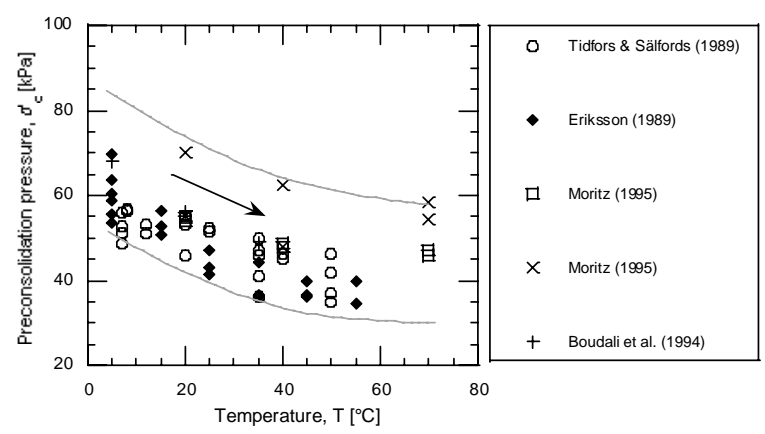

Figure 7. Influence of temperature on the preconsolidation pressure. 
The thermal strain rate tensor, $\dot{\varepsilon}^{T}$, due to thermo-mechanical loading can be split into thermo-elastic, $\dot{\varepsilon}^{e, T}$, and thermo-plastic, $\dot{\varepsilon}^{p, T}$, components :

$$
\dot{\varepsilon}_{i j}^{T}=\dot{\varepsilon}_{i j}^{e, T}+\dot{\varepsilon}_{i j}^{p, T}
$$

\subsection{Thermo-elasticity}

The thermo-elastic strain, $\varepsilon_{i j}^{e T}$, is composed of the superposition of a mechanical elastic strain under adiabatic conditions, $\varepsilon_{i j}^{e, m}$, and a reversible thermal strain, $\varepsilon_{i j}^{e, T}$ :

$$
\dot{\varepsilon}_{v}^{e, T}=\beta_{s}^{\prime} \dot{T}
$$

where $\dot{\varepsilon}_{v}^{e, T}$ is the thermo-elastic volumetric strain rate $\left(\dot{\varepsilon}_{v}^{e, T}=\operatorname{tr}\left(\dot{\varepsilon}_{i j}^{e, T}\right)\right)$.The thermal expansion coefficient of the solid skeleton, $\beta_{s}^{\prime}$, has to be explicated : here it is the skeleton coefficient, which differs from the saturating fluid one. Experimentally, one can measure the drained skeleton coefficient, the water coefficient, the grain coefficient, the undrained bi- or tri-phases material, or any other mixture, depending on the stress and pore pressure control. $\beta_{s}^{\prime}$, varies strongly with temperature and slightly with pressure according to:

$$
\beta_{s}^{\prime}=\left(\beta_{s 0}^{\prime}+\zeta T\right) \xi
$$

in which $\beta_{s 0}^{\prime}$ is the isotropic thermal expansion coefficient at a reference temperature, $T_{0}$ (usually ambient temperature), and $\xi$ the ratio between the critical state pressure for the initial state, $p_{c o}^{\prime}$, and the mean effective pressure, $p^{\prime}$, at ambient temperature:

$$
\xi=\frac{p_{c 0}^{\prime}}{p^{\prime}}
$$

$\zeta$ corresponds to the slope of the variation of $\beta_{s}^{\prime}$ with respect to the current temperature, $T$, at $\xi=1$.

\subsection{Thermo-plasticity}

Generally speaking, the yield surface for an isotropic loading may be written as:

$$
p_{0}=F_{1}(T) \mathrm{F}_{2}\left(\beta \varepsilon_{v}^{p}\right)
$$

This means that two isotropic hardening mechanisms exist, a thermal one and a mechanical / strain one. These processes are not coupled but only combined through 
a multiplicative function. The mechanical hardening takes the classical form for cohesive soils, as indicated in oedometer tests:

$$
p_{0}=F_{1}(T) \exp \left\{\beta \varepsilon_{v}^{p}\right\}=p_{c 0}(T) \exp \left\{\beta \varepsilon_{v}^{p}\right\}
$$

$p_{c 0}(T)$ is the value of the preconsolidation pressure at temperature $T$, $\beta=\frac{\lambda-\kappa}{1+e}$ is the plastic compressibility modulus. A dependency law for the thermal evolution of the preconsolidation pressure, $p_{c 0}(T)$, is introduced to take into account the thermal effect on the yield limit (Laloui and Cekerevac, 2003):

$$
p_{c 0}(T)=p_{c 0}\left(T_{0}\right)\left\{1-\gamma_{T} \log \left[T / T_{0}\right]\right\}
$$

where $p_{c 0}\left(T_{0}\right)$ is the value of the preconsolidation pressure at the reference temperature and $\gamma_{T}$ a material parameter. The expression of the isotropic thermoplastic yield limit is thus given by:

$$
f_{T i}=p^{\prime}-p_{c 0}\left(T_{0}\right) \exp \left\{\beta \varepsilon_{v}^{P}\right\}\left\{1-\gamma_{T} \log \left[T / T_{0}\right]\right\}
$$

Equation [22] contains two material parameters: $\beta$ expressing the evolution of mechanical hardening and $\gamma_{T}$ controlling the evolution of thermal hardening. This latter one defines the shape of the yield limit as shown in Figure 8.
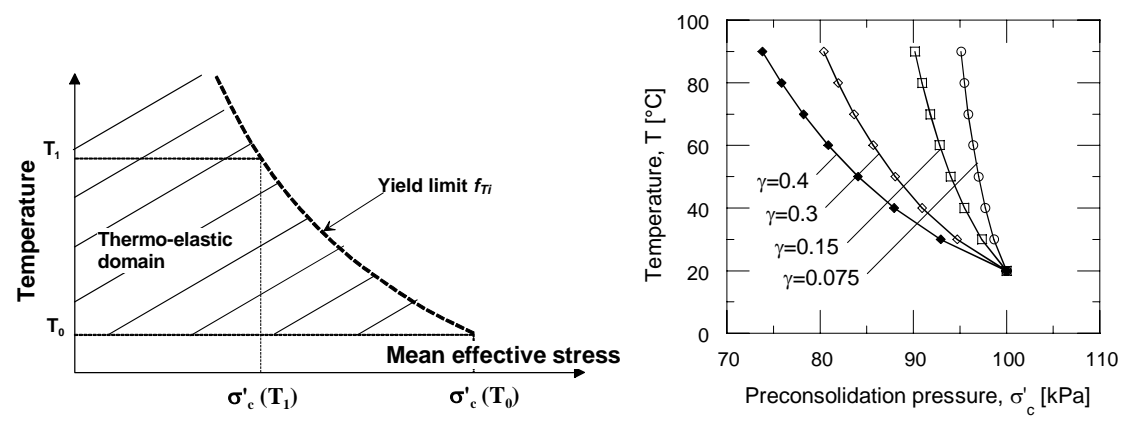

Figure 8. Isotropic thermo-plastic yield limit and its dependency on the parameter $\gamma_{T}$.

The flow rule is associated:

$$
f_{T i}\left(p, T, \varepsilon_{v}^{p}\right)=g_{T i}\left(p, T, \varepsilon_{v}^{p}\right)
$$




$$
\dot{\varepsilon}_{i i}^{p}=\lambda_{i} \frac{\partial g_{T i}}{\partial \sigma_{i i}^{\prime}}=\frac{\lambda_{i}}{3}
$$

The plastic multiplier, $\lambda_{i}$, is determined using the consistency equation.

Some recent contributions (Cekerevac and Laloui, 2004; Laloui and François, 2009; Hueckel et al., 2009) clarified the friction angle dependency on temperature and the various thermal effects on the failure limit.

\subsection{Heat transfers}

Heat transfer in solids follows a Fourier's like model for conduction. The conduction heat flux $q$ writes:

$$
\underline{q}^{\text {cond }}=-\lambda \underline{\nabla}(T)
$$

where $T$ denotes the temperature. The conductibility is noted $\lambda$. It depends on the saturation degree and can be evaluated based on a kind of mixture formula between the phases (solid and fluids). It depends on the temperature:

$$
\lambda=\lambda(T)
$$

In materials saturated by one or two moving fluids, heat is also transported by fluid (flux $q^{(i)}$ ), this is the advection process.

$$
\underline{q}^{\operatorname{ad}(i)}=H \underline{q}^{(i)}
$$

$H$ is the fluid enthalpy, which depends on the temperature and on the saturation degree. The total heat flux is then:

$$
\underline{q}^{\text {tot }}=\underline{q}^{\text {cond }}+\underline{q}^{a d(1)}+\underline{q}^{\text {ad(2) }}
$$

The heat balance equation writes classically:

$$
\operatorname{div}\left(\underline{q}^{t o t}\right)+\frac{\partial H}{\partial t}=0
$$

Heat transfer in soils and rocks is generally not as nonlinear as the fluid flow or the solid mechanics. Then numerical convergence is easier. Moreover the coupling of mechanics and fluid flow on heat transfer is lower then the coupling in the opposite direction. Heat transfer has then to be analysed first. 


\section{Generic structure for constitutive models dedicated to multi-physics coupling.}

The two preceding sections have detailed the basic physical processes of successively partial saturated geomaterials mechanics and thermo-mechanics. Some similarities have been pointed. One could now elaborated similar developments for e.g chemo-mechanical behaviour. In this section, we intend to give a general and detailed framework of any coupled multiphysics constitutive model.

\subsection{Variables and notations}

In the following, $\varepsilon_{i j}$ denotes the strain tensor and $\sigma_{i j}$ the stress tensor. We will consider only one coupling, between the mechanical behaviour and one environmental phenomenon: temperature $(T)$, suction $(s)$, or chemistry $(c)$, denoted generically $\gamma$, which is always a scalar.

For simplicity, the mechanical behaviour is based on the Cam-Clay concept, inserted in a cap model. Elastoplasticity is considered, i.e. strain supports an additive decomposition into reversible - elastic and irreversible - plastic parts:

$$
\dot{\varepsilon}_{i j}=\dot{\varepsilon}_{i j}^{e}+\dot{\varepsilon}_{i j}^{p}
$$

Strain variations are induced by either stress variation (denoted by $m$ exponent) or by a variation of the environmental variable $\gamma$ (denoted by $\gamma$ exponent). The strain rate writes:

$$
\begin{gathered}
\dot{\varepsilon}_{i j}=\dot{\varepsilon}_{i j}^{m}+\dot{\varepsilon}_{i j}^{\gamma} \\
\dot{\varepsilon}_{i j}^{m}=\dot{\varepsilon}_{i j}^{e, m}+\dot{\varepsilon}_{i j}^{p, m} \\
\dot{\varepsilon}_{i j}^{\gamma}=\dot{\varepsilon}_{i j}^{e, \gamma}+\dot{\varepsilon}_{i j}^{p, \gamma}
\end{gathered}
$$

Internal variables are the stress tensor, $\sigma_{i j}$, the coupling variable, $\gamma$, and memory variables as the void ratio, $e$, and/or the plasticity threshold, like the preconsolidation pressure, $p_{0}$. The stresses and the coupling variable play the same role in the model elaboration. In order to simplify the writing of the time integration algorithms for the constitutive model, (Vaunat et al., 2000) suggest the use of generalized variables:

$$
\begin{gathered}
\underline{\sigma}^{*}=\left(\bar{\sigma}_{11}, \bar{\sigma}_{22}, \bar{\sigma}_{33}, \bar{\sigma}_{12}, \bar{\sigma}_{13}, \bar{\sigma}_{23}, \gamma\right)^{t} \\
\underline{\varepsilon}^{*}=\left(\varepsilon_{11}, \varepsilon_{22}, \varepsilon_{33}, \varepsilon_{12}, \varepsilon_{13}, \varepsilon_{23}, \varepsilon_{v}^{\gamma}\right)^{t}
\end{gathered}
$$

where $\varepsilon_{i j}$ are the total strains and $\varepsilon_{v}^{\gamma}$ is the volumetric strain induced by the coupling variable rate. It is commonly considered that the coupling only induces volumetric strains and not deviatoric ones. Moreover, total strain rates are considered (and not only purely mechanical induced strains) as they are the basic input in time integration scheme in finite element codes. 


\subsection{Elasticity}

The elasticity constitutive model for the mechanical part writes:

$$
\dot{\sigma}_{k l}=C_{k l i j}^{e} \dot{\varepsilon}_{i j}^{m, e}
$$

With the elasticity tensor $C_{k l i j}^{e}$ defined as:

$$
C_{k l i j}^{e}=2 G \delta_{i k} \delta_{j l}+\left[\frac{(1+e)}{3 \kappa} \sigma_{m m}-\frac{2}{3} G\right] \delta_{i j} \delta_{k l}
$$

where $\delta_{i j}$ is the Kronecker symbol, $G$ the shear modulus, $\kappa$ the volumetric elastic modulus (logarithmic law). The material parameters $G$ and $\kappa$ may depend on the coupling variable $\gamma$. The elastic strain induced by the coupling variable $\gamma$ rate is a purely volumetric one:

$$
\dot{\varepsilon}_{i j}^{e, \gamma}=K \dot{\gamma} \delta_{i j}
$$

It may be a non-linear relation, i.e. $K$ may depend on the stress and coupling variable states. This model indicates that changes related to chemistry, temperature, or suction may induce micro-structural changes, and, ions and water molecules movements. The elastic laws may rewrite:

$$
\begin{gathered}
\underline{\dot{\sigma}}=\underline{\underline{C}}^{e} \underline{\dot{\varepsilon}}^{e, m}=\underline{\underline{C}}^{e} \cdot\left(\underline{\dot{\varepsilon}}^{e}-\dot{\varepsilon}_{v}^{e, \gamma} \frac{\underline{I}}{3}\right)=\underline{\underline{C}}^{e} \underline{\dot{\varepsilon}}^{e}-\chi \dot{\varepsilon}_{v}^{\gamma, e} \underline{I} \\
\dot{\gamma}=\frac{1}{K} \dot{\varepsilon}_{v}^{e, \gamma}
\end{gathered}
$$

with $\chi$ the volumetric elasticity modulus. Considering the generalized variables [32]:

$$
\underline{\dot{\sigma}}^{*}=\underline{\underline{C}}^{* e} \cdot \dot{\underline{\varepsilon}}^{* e}
$$

with the following elasticity matrix (3D problems):

$$
\stackrel{C^{*} e}{=}\left[\begin{array}{ccccccc}
\chi+\frac{4}{3} G & \chi-\frac{2}{3} G & \chi-\frac{2}{3} G & 0 & 0 & 0 & -\chi \\
\chi-\frac{2}{3} G & \chi+\frac{4}{3} G & \chi-\frac{2}{3} G & 0 & 0 & 0 & -\chi \\
\chi-\frac{2}{3} G & \chi-\frac{2}{3} G & \chi+\frac{4}{3} G & 0 & 0 & 0 & -\chi \\
0 & 0 & 0 & 2 G & 0 & 0 & 0 \\
0 & 0 & 0 & 0 & 2 G & 0 & 0 \\
0 & 0 & 0 & 0 & 0 & 2 G & 0 \\
0 & 0 & 0 & 0 & 0 & 0 & 1 / K
\end{array}\right]
$$




\subsection{Plasticity threshold}

The yield surface has to take into account three types of mechanical behaviours:

- Nearly isotropic compression, characterised by a pre-consolidation pressure as plasticity threshold $p_{0}$;

- Deviatoric shear failure based on internal friction model and friction angle $\phi$ or $p-q$ slope $M$;

- $\quad$ Traction strength $\sigma_{t}$, isotropic traction $p_{s}$ or cohesion $c$.

These three parameters $p_{0}, M$ and $\sigma_{t}$ may depend on the coupling variable $\gamma$.

A schematic view of such a yield surface is given in figure 9-a. In the $p-q$ invariant plane, it is composed of an elliptic cap, a friction line and a traction cut-off.

(a)

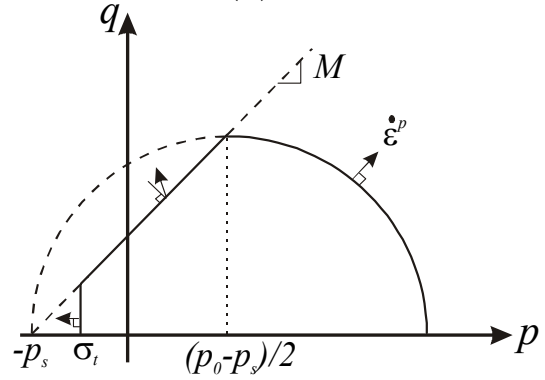

(b)

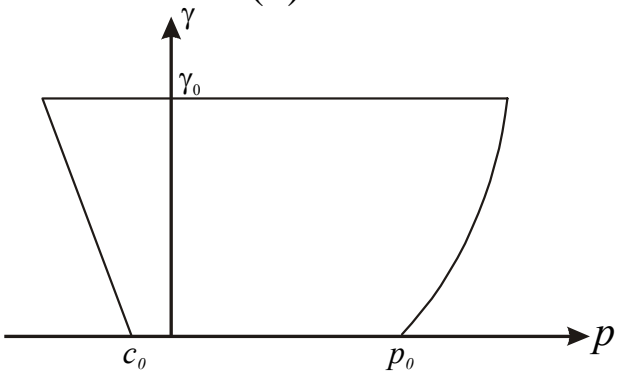

Figure 9. Yield surface for purely mechanical problems (a) and for Mechanicalenvironmental coupling (b).

This yield surface writes:

$$
\begin{aligned}
& f_{1}=q^{2}+M^{2}\left(p+p_{s}\right)\left(p-p_{0}\right)=0 ; p \geq\left(p_{0}-p_{s}\right) / 2 \\
& f_{2}=q-M\left(p+p_{s}\right)=0 ; \sigma_{\mathrm{t}}<p \leq\left(p_{0}-p_{s}\right) / 2 \\
& f_{3}=p+\sigma_{t}=0
\end{aligned}
$$

The slope $M$ may depend on the Lode angle, as proposed e.g. by (Van Eekelen, 1980).

Additionally, a specific plasticity threshold may be written for the coupling variable:

$$
f_{4}=\gamma-\gamma_{0}=0
$$


The mechanical - environmental coupling is schematised for the yield surface in the figure 9-b, where the coupling between the environmental variable $\gamma$ and respectively the pre-consolidation pressure $p_{0}$, and the traction strength $\sigma_{t}$.

\subsection{Plastic strains}

The plastic strain rate derives from a flow potential which often differs from the yield surface (non-associative plasticity) in the stress plane (figure 9-a) but generally coincides with the yield surface in the coupling plane (figure 9-b).

Once again the coupling term is a purely volumetric one. With the generalized variables, this writes:

$$
\underline{\dot{\varepsilon}}^{* p}=\underline{\dot{\varepsilon}}^{p, m}+\dot{\varepsilon}_{v}^{p, \gamma} \frac{\underline{I}}{3}=\dot{\lambda}^{p} \frac{\partial g}{\partial \underline{\sigma}^{*}}
$$

with :

$$
\frac{\partial g}{\partial \underline{\sigma}^{*}}=\left(\frac{\partial g}{\partial \sigma_{1}}+\frac{1}{3} \frac{\partial g}{\partial \gamma}, \frac{\partial g}{\partial \sigma_{2}}+\frac{1}{3} \frac{\partial g}{\partial \gamma}, \frac{\partial g}{\partial \sigma_{3}}+\frac{1}{3} \frac{\partial g}{\partial \gamma}, \frac{\partial g}{\partial \sigma_{4}}, \frac{\partial g}{\partial \sigma_{5}}, \frac{\partial g}{\partial \sigma_{6}}, \frac{\partial g}{\partial \gamma}\right)
$$

\subsection{Application to different coupling phenomena}

Generally speaking, it appears the following environmental dependences of the plastic material parameters, more precisely the yield surface parameters:

- The pre-consolidation pressure $p_{0}$ is highly dependent on the environmental phenomena;

- The friction angle at the critical state $\phi$ seems quite independent on the environmental phenomena;

- $\quad$ The cohesion $c$ and the traction strength $\sigma_{t}$ depend on the suction and on the chemistry (damage, weathering of cementation bridges). However the know-how on these aspects are not highly developed.

Following these observations, most papers have been devoted to the cap of the yield surface, i.e. the Cam-Clay equation $f_{l}$ (see Equation 38), and to the preconsolidation pressure environmental evolution. The hardening law will govern this evolution. It depends on two internal variables:

$$
p_{0}=\Phi\left(p_{0}^{*}, \gamma\right)
$$

where $p_{0}{ }^{*}$ is the $p_{0}$ value when the coupling variable $\gamma$ vanishes.

Classically, $p_{0}{ }^{*}$ evolves with the volumetric plastic strain, following the CamClay concept: 


$$
\dot{p}_{0}^{*}=\frac{1+e}{\lambda-\kappa} p_{0}^{*} \dot{\varepsilon}_{v}^{p}
$$

Deriving equation [43] with respect to the time:

$$
\dot{p}_{0}=\frac{\partial \Phi}{\partial p_{0}^{*}} \dot{p}_{0}^{*}+\frac{\partial \Phi}{\partial \gamma} \dot{\gamma}=\frac{\partial \Phi}{\partial p_{0}^{*}} \frac{1+e}{\lambda-\kappa} p_{0}^{*} \dot{\varepsilon}_{v}^{p}+\frac{\partial \Phi}{\partial \gamma} \dot{\gamma}
$$

The preconsolidation pressure rate depends on the plastic volumetric strain $\varepsilon_{v}^{p}$ and on the coupling variable $\gamma$. If one considers a plastic loading where the stresses do not vary, following the consistency condition:

$$
f=\frac{\partial f}{\partial \sigma_{i j}} \dot{\sigma}_{i j}+\frac{\partial f}{\partial p_{0}} \dot{p}_{0}=0
$$

and

$$
\dot{p}_{0}=0
$$

Then the plastic strain rate induced by a purely environmental loading writes:

$$
\dot{\varepsilon}_{v}^{p}=\frac{-\frac{\partial \Phi}{\partial \gamma} \dot{\gamma}}{\frac{\partial \Phi}{\partial p_{0}^{*}} \frac{1+e}{\lambda-\kappa} p_{0}^{*}}
$$

\section{Model application to suction, thermal or chemical coupling}

In the following development, $s$ denotes the suction in the hydraulic partly saturated coupling, $T$ the temperature for the heat coupled model and $c$ a measure of the concentration for the chemical coupled model. The same letters will be used as indices for denoting the variables, in place of the generic notation $\gamma$. The considered models here are based, respectively,

- for the hydraulic partly saturated coupling, on the Barcelona Basic Model (Alonso et al., 1990)

- $\quad$ for the chemical coupling on the Hueckel model (Hueckel, 1997)

- for the thermal coupling on the Laloui and Cekerevac model (Laloui and Cekerevac, 2003).

\subsection{Elastic strains}

In this section we describe the reversible strain induced by the environmental load. Such elastic strain may be expressed simply as proportional to the variation of the considered load (as for the chemical load). However, for suction and temperature 
effects, the reversible strain is no more linear and complementary dependency laws are needed.

\subsubsection{Suction coupling}

A suction increase induces a strain contraction, which is often described with a logarithmic incremental law (figure 10-a):

$$
\dot{\varepsilon}_{v}^{s, e}=\frac{1}{3} \frac{\kappa_{s}}{(1+e)} \frac{\dot{s}}{\left(s+p_{a t}\right)}
$$

where $\kappa_{s}$ is a compressibility constant parameter and $p_{a t}$ denotes the atmospheric pressure.

\subsubsection{Chemical coupling}

A chemical concentration increase induces a volumetric dilatation (figure 10-b):

$$
\varepsilon_{v}^{c, e}=F_{0} \exp \left[\beta_{0}(1-c+\ln c)\right]
$$

where $F_{0}$ denotes the maximal strain, for a concentration equal to unity. $\beta_{0}$ is the curve slope for half of the maximum strain $F_{0} / 2$. An incremental formulation follows:

$$
\begin{aligned}
& \dot{\varepsilon}_{i j}^{c, e}=-\frac{1}{3} \beta \dot{c} \delta_{i j} \\
& \beta=-F_{0} \beta_{0} \exp \left[\beta_{0}(1-c+\ln c)\right]\left(\frac{1}{c}-1\right)
\end{aligned}
$$

(a)

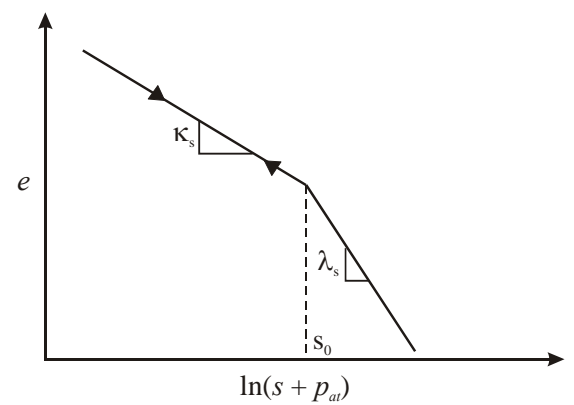

(b)

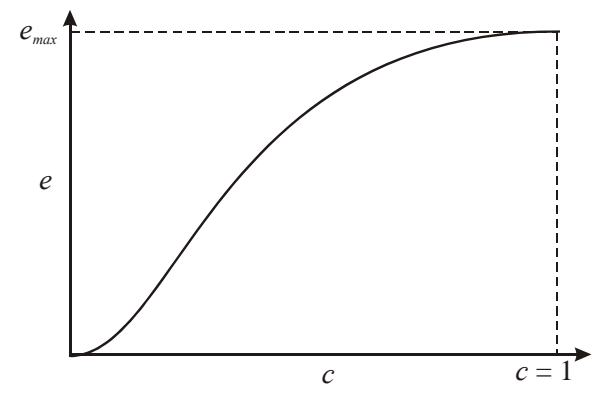

Figure 10. Elastic behaviour induced by a change of, (a) suction, (b) concentration.

\subsubsection{Thermal coupling}

The temperature increase induces a non linear elastic dilatation: 


$$
\varepsilon_{v}^{T, e}=\beta_{s}\left(T-T_{0}\right)
$$

where $\varepsilon_{v}^{T, e}$ is the volumetric thermo-elastic strain, $\beta_{s}$ the volumetric thermal dilation coefficient and $T-T_{0}$ the thermal variation.

\subsection{Plasticity yield surface}

The yield surface depends on 3 parameters: the pre-consolidation pressure, $p_{0}$, the friction angle, $\phi$, and the cohesion, $c$.

\subsubsection{Suction coupling}

In the Barcelona Basic Model, the preconsolidation pressure is governed by:

$$
p_{0}=p_{c}\left(\frac{p_{0}^{*}}{p_{c}}\right)^{\frac{\lambda(0)-\kappa}{\lambda(s)-\kappa}}
$$

Basically, drying a soil increases its strength. Other shape may be used (e.g. for chalk, see (Collin, 2003). The friction angle is generally considered as quite independent from the suction. In the Barcelona Basic Model, the cohesion model is linear:

$$
c=c_{0}+k \cdot s
$$

\subsubsection{Chemical coupling}

In the Hueckel Model (Hueckel, 1997), the preconsolidation pressure is governed by:

$$
p_{0}=p_{0}^{*} S(c) ; \quad S(c)=\exp (-a c)
$$

The strength is reduced by an increase in chemical concentration. The friction angle is generally considered as quite independent from the chemistry. Few exist about the cohesion. Cohesion could be weathered by concentration increase (Nova and Parma, 2005).

\subsubsection{Thermal coupling}

In the Laloui and Cekerevac Model (Laloui and Cekerevac, 2003), the apparent preconsolidation pressure decreases with the temperature increase:

$$
p_{0}(T)=p_{0}^{*}\left(T_{0}\right)\left\{1-\gamma_{T} \log \left[T / T_{0}\right]\right\}
$$

$\gamma_{T}$ being a material parameters (Laloui et al., 2005c). The friction angle may be considered as quite independent from the temperature (Cekerevac and Laloui, 2004). The plastic thermal effect induces a more ductile behaviour. 


\subsubsection{Coupled multiphysical processes}

Few models handle the coupling processes between mechanics and more than one environmental variable. Among them, one can refers to the thermo-plastic model for unsaturated soils, ACMEG-TS, by François and Laloui (2008).

\section{Finite element for coupled processes}

\subsection{Finite element modelling: monolithical approach}

Modelling the coupling between different phenomena should imply to model each of them and, simultaneously, all the interactions between them. A first approach consists in developing new finite elements and constitutive laws especially dedicated to the physical coupled problem to be modelled. This approach allows taking accurately all the coupling terms into account. However there are some drawbacks that will be discussed in a later section.

The number of basic unknowns and following the number of degrees of freedom - dof per node are increased. This has a direct effect on the computer time used for solving the equation system (up to the third power of the total dof number). Coupled problems are highly time consuming. Isoparametric finite element will often be considered. However some specific difficulties may be encountered for specific problems. Nodal forces or fluxes are computed in the same way as for decoupled problems. However stiffness matrix evaluation is much more complex, as interactions between the different phenomena are to be taken into account. Remember that the Newton - Raphson stiffness or iteration matrix is the derivative of internal nodal forces / fluxes with respect to the nodal unknowns (displacements / pressures / temperature...). The complexity is illustrated by the following scheme of the stiffness matrix, restricted to the coupling between two problems:

\begin{tabular}{|l|l|}
\hline $\begin{array}{l}\text { Derivative of problem } \mathbf{1} \text { nodal forces } \\
\text { with respect to problem } \mathbf{1} \text { nodal } \\
\text { unknowns }\end{array}$ & $\begin{array}{l}\text { Derivative of problem } \mathbf{1} \text { nodal forces } \\
\text { with respect to problem } \mathbf{2} \text { nodal } \\
\text { unknowns }\end{array}$ \\
\hline $\begin{array}{l}\text { Derivative of problem } \mathbf{2} \text { nodal forces } \\
\text { with respect to problem } \mathbf{1} \text { nodal } \\
\text { unknowns }\end{array}$ & $\begin{array}{l}\text { Derivative of problem } \mathbf{2} \text { nodal forces } \\
\text { with respect to problem } \mathbf{2} \text { nodal } \\
\text { unknowns }\end{array}$ \\
\hline
\end{tabular}

The part of the stiffness matrix in cells 1-1 and 2-2 are similar or simpler to the ones involved in uncoupled problems. The two other cells 1-2 and 2-1 are specific to coupling and may be of certain complexity. Remember also that the derivative consider internal nodal forces / fluxes as obtained numerically, i.e. taking into account all numerical integration / derivation procedures. On the other hand, large difference of orders of magnitude between different terms may cause troubles in solving the problem and so need to be checked. 
Numerical convergence of the Newton - Raphson process has to be evaluated carefully. It is generally based on some norms of the out-of-balance forces / fluxes. However, coupling implies often mixing of different kinds of dof, which may not be compared without precaution. Convergence has to be obtained for each basic problem modelled, not only for one, which would then predominate in the computed indicator.

\subsection{Physical aspects: various terms of coupling}

A large number of different phenomena may be coupled. It is impossible to discuss here all potential terms of coupling, and we will restrict ourselves to some basic cases. In the following paragraphs, some fundamental aspects of potential coupling are briefly described.

\subsubsection{Hydromechanical coupling}

Number of dof per node: 3 (2 displacements +1 pore pressure) for 2D analysis and 4 ( 3 displacements +1 pore pressure) for 3D analysis.

Coupling mechanical deformation of soils or rock mass and one fluid flow in pores is a frequent problem in geomechanics. The first coupling terms are related to the influence of pore pressure on mechanical equilibrium through the Terzaghi's postulate

$$
\underline{\sigma}=\underline{\sigma^{\prime}}+p \underline{I}
$$

with the effective stress tensor $\sigma^{\prime}$ related to the strain rate tensor thanks to the constitutive equation, and the unity tensor $\underline{I}$.

The second type of coupling concerns the influence of the solid mechanics behaviour on the flow process, which comes first through the storage term. Storage of water in saturated media is mainly due to pores strains, i.e. to volumetric changes in soil / rock matrix:

$$
\dot{S}=\dot{\varepsilon}_{v}
$$

Another effect, which may be considered, is the permeability change related to the pore volume change, which may for example be modelled by the Kozeni Carman law as a function of the porosity $k=k(n)$. This effect is much more significant for fractures (see next paragraph).

The time dimension may cause some problems. First implicit scheme are used for the solid mechanics equilibrium and various solutions are possible for the pore pressure diffusion process. Consistency would imply to use fully implicit schemes for the two problems. Moreover, it has been shown that time oscillations of the pore pressure may occur for other time schemes. Associated to the Terzaghi's postulate, 
oscillations could appear also on the stress tensor, what can quickly degrade the numerical convergence rate for elastoplastic or elastoviscoplastic constitutive laws.

When using isoparametric finite elements, the shape function for geometry and for pore pressure are identical. Let us consider for example a second order finite element. As the displacement field is of second order, the strain rate field is linear. For an elastic material, the effective stress tensor rate is then also linear. However the pore pressure field is quadratic. Then the Terzaghi's postulate mixes linear and quadratic field, which is not highly consistent. Some authors have then proposed to mix in one element quadratic shape functions for the geometry and linear shape functions for pore pressure. But then problems arrive with the choice of spatial integration points (1 or 4 Gauss points?).

Numerical locking problems may also appear for isoparametric finite element when the two phases material (fluid + rock) is quite incompressible, i.e. for very short time steps with respect with the fluid diffusion time scale. Specific elements have to be developed for such problems.

\subsubsection{Two fluids flow in rigid porous media coupling}

Flow in partly saturated rigid media is here considered. Flow in oil or gas reservoirs two or three fluids among oil, gas, condensates and water. Partial solving or mixture between different fluids is sometimes possible. Then two or more dof per node are to be considered. The permeability and storage equation of each phase are depending on the suction or saturation level, and so the problem may be highly nonlinear. However, coupling is not difficult to numerically be developed, as the formulation is similar for each phase.

\subsubsection{Diffusion and transport coupling}

Heat and one fluid flow in a rigid porous media are concerned here. The fluid specific weight and viscosity is depending on the temperature, and the heat transport by advection - diffusion process is depending on the fluid flow. Then a diffusion process and an advection - diffusion process have to be solved simultaneously.

Number of dof per node: 2 (fluid pore pressure and temperature).

\subsubsection{Thermo-hydro-mechanical coupling}

The phenomena considered here are much more complex as they associate multiphase fluid flow, hydromechanical coupling and temperature effects. All the features described in the preceding sections are to be considered here, associated to some new points. 
Heat diffusion has to be modelled. Temperature variation affects fluid flow, by a modification of the fluid specific weight or viscosity. Moreover, if the two fluids concerned are a liquid and a gas (e.g. water and air), then equilibrium between the phases has to be modelled: dry air - vapour equilibrium.

Heat transfer is governed not only by conduction but also by advection by the liquid and gas movements. Similarly transfers of vapour and gas species in the gas phase are governed by diffusion and gradient of species density, but also by advection with the global gas movements. If the concerned geomaterial has a very low permeability (like clay), then the diffusion effects will predominate and advection doesn’t necessitate specific formulation (Collin et al., 1999).

Finally the total number of dof per node is 5 for a 2D problem: 2 displacements, 2 fluid pore pressures and the temperature.

\subsection{Finite element modelling: staggered approach}

Monolithical approach of coupled phenomena implies identical space and time meshes for each phenomenon. This is not always possible, for various reasons. The coupled problems may have different numerical convergence properties, generally associated to different physical scales or non-linearity. For example, a coupled hydromechanical problem may need large time steps for the fluid diffusion problem, in order to allow, in each step, fluid diffusion along distance of the order of magnitude of the finite elements. In the same time, strong non-linearity may occur in solid mechanics behaviour (strong elastoplasticity changes, interface behaviour, strain localisation...) and then the numerical convergence needs short time - loading steps, which should be adapted automatically to the rate of convergence. Then it is quite impossible to obtain numerical convergence for identical time and space meshes.

Coupled problems are generally presenting a higher non-linearity level then uncoupled ones. Then inaccuracy in parameters or in the problem idealisation may cause degradations of the convergence performance.

How can we solve such problems and obtain a convincing solution? First of all, a good strategy would be to start with the uncoupled modelling of the leading process, and to try to obtain a first not too bad solution. Then one can add a first level of coupling and complexity, followed by a second one... until the full solution is obtained.

However such trick is not always sufficient. Staggered approaches may then give an interesting solution. In a staggered scheme, the different problems to be coupled are solved separately, with (depending on the cases) different space or time mesh, or different numerical codes. However, the coupling is ensured thank to transfer of 
information between the separated models at regular meeting points. This concept is summarised in figure 11. It allows theoretically coupling any models together.

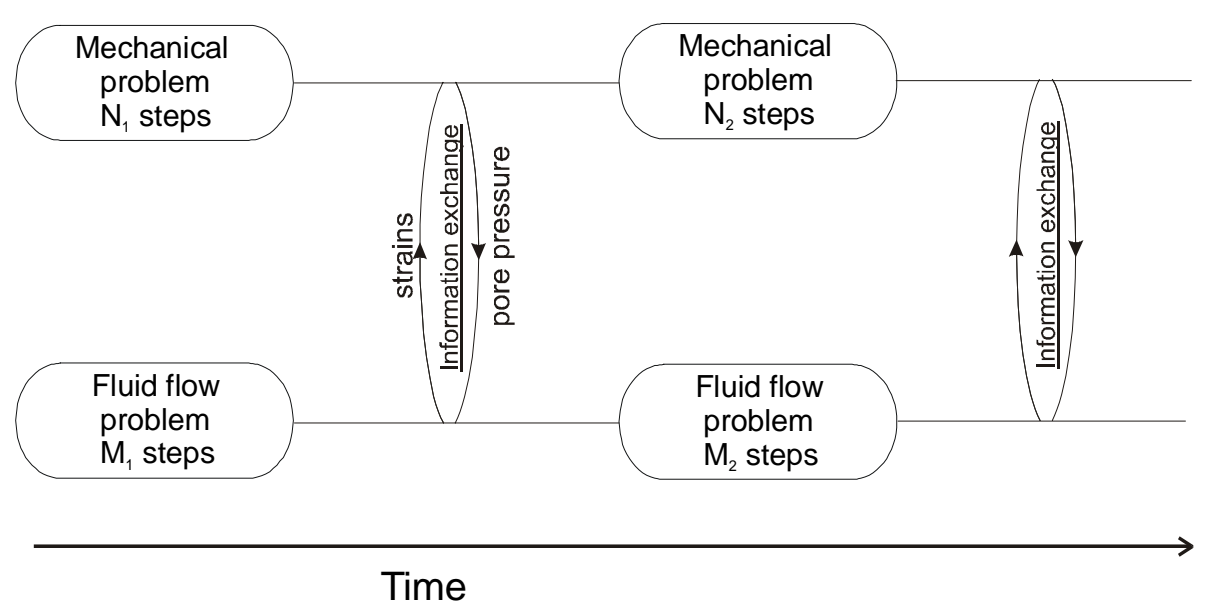

Figure 11. Scheme of a staggered coupling

When using different spatial meshes, or when coupling finite elements and finite differences codes, the transfer of information needs often an interpolation procedure, as the information to be exchanged are not defined at the points in the different meshes.

The accuracy of the coupling scheme will mainly depend on the information exchanges frequency (which is limited by the lower time step that can be used) and by the type of information exchanged. The stability and accuracy of the process has been checked by different authors (Turska et al., 1993, Zienckiewicz et al., 1988). It has been shown that a good choice of the information exchange may improve highly the procedure efficiency.

\section{Acknowledgements}

Authors are grateful to FRS-FNRS, La Communauté Française de Belgique and the European Commission for the financial help to their research projects. 
Multi-physical processes in geomechanics 25

\section{References}

Alonso E.E., Gens A., Josa A., “A constitutive model for partially saturated soils”, Géotechnique, vol. 40 n. 3, 1990, p. 405-430.

Biot M.A., “General theory of three-dimensional consolidation”, J. Appl. Phys., vol. 12, 1941, p. $155-164$.

Bishop A.W., “The principle of effective stress”, Tecnisk Ukeblad, vol. 39, 1959, p. 859-863.

Boukpeti N., Charlier R., Hueckel T., “Modelling contamination of clays”, VII International conference on Computational Plasticity, Owen DRJ, Onate E, Suarez B (eds), Barcelona, 2003, 19 p.

Charlier R., Radu J.P., Collin F., "Numerical modelling of coupled transient phenomena”, ALERT autumn school 2001, Revue Française de Génie Civil, vol. 5, n 6, 2001.

Charlier R., Laloui L., Collin F., "Numerical modelling of coupled poromechanics processes”, ALERT Autumn School 2006, Revue Européenne de Génie Civil, vol. 10, n 6-7, 2006, p. 669-7001.

Cekerevac C., Thermal effects on the mechanical behaviour of saturated clays: an experimental and numerical study, Doctoral thesis No 2828, Swiss Federal Institute of Technology, Lausanne; 2003.

Cekerevac C., Laloui L., "Experimental study of the thermal effects on the mechanical behaviour of a clay", International Journal of Numerical and Analytical Methods in Geomechanics, vol. 28, 2004, p. 209-228.

Collin F., Li X.L., Radu J.P., Charlier R., “Clay barriers assessment : a coupled mechanical and moisture transfer model”, Proc. ECCM'99, European Conference on Computational Mechanics, Munich (Sept. 1999).

Collin F., Cui Y.J., Schroeder C., Charlier R., "Mechanical behaviour of Lixhe chalk partly saturated by oil and water: experiment and modeling”, Numerical and Analytical Methods in Geomechanics, vol. 26, 2002, p. 897-924.

Collin F. Couplages thermo-hydro-mécaniques dans les sols et les roches tenders partiellement saturés. Doctoral Thesis, University of Liège, 2003.

Collin F., Laloui L., Charlier R.. "Unified approach of coupled constitutive laws”. ALERT autumn school 2005, Revue Européenne de Génie Civil, vol. 9, n5-6, 2005.

Coussy O., Mechanics of Porous Continua, London, Wiley, 1995.

Detournay E., Cheng A.H.D., Fundamentals of poroelasticity. Chapter 5 in Comprehensive Rock Engineering, Vol. II, p. 113-171, 1993.

Fleureau J M., Influence d'un champ thermique ou électrique sur les phénomènes d'interaction solide-liquide dans les milieux poreux, Doctoral thesis, Ecole Centrale de Paris, 1979.

François B., L. Laloui. "ACMEG-TS: A constitutive model for unsaturated soils under nonisothermal conditions". International Journal of Numerical and Analytical Methods in Geomechanics, vol. 32, 2008, p. 1955-1988. 
Fredlund D.G., Morgenstern N. R., "Stress state variables for unsaturated soils”, Journal of the geotechnical engineering division, ASCE, 103(GT5), 1977, p. 447-466.

Gens A., Charlier R., “Environmental geomechanics”, ALERT Autumn School 2001, Revue Française de Génie Civil, vol. 5, n6, 2001.

Hueckel T., Borsetto M., "Thermoplasticity of saturated soils and shales: Constitutive equations”, Journal of Geotechnical Engineering, vol. 116, n 2, 1990, p. 1765-1777.

Hueckel T., "Chemo-plasticity of clays subjected to stress and flow of a single contaminant", International Journal for Numerical and Analytical Methods in Geomechanics, vol. 21, 1997, p. 43-72.

Hueckel T., François B., Laloui L. "Explaining thermal failure in saturated clays", Géotechnique, vol. 59, n³, pp. 197-212, doi: 10.1680/geot.2009.59.3.197, 2009.

Laloui L., Modélisation du comportement thermo-hydro-mécanique des milieux poreux anélastiques. Doctoral Thesis, Ecole Centrale Paris, 1993.

Laloui L., Geiser F., Vulliet L., “Constitutive modeling of unsaturated soils”, ALERT autumn school 2001, Revue française de genie civil, vol. 5, 2001, p. 797-807.

Laloui L., Cekerevac C., “Thermo-plasticity of clays: an isotropic yield mechanism”, Computers and Geotechnics, vol. 30, n 8, 2003, p. 649-660.

Laloui L., Charlier R., Pijaudier-Cabot G., "Coupled multi-physics processes in geomechanics”, ALERT Autumn School 2005, Revue Européenne de Génie Civil, vol. 9, $n^{\circ} 5-6,2005$.

Laloui L., Nuth M., "An introduction to the constitutive modelling of unsaturated soils", ALERT autumn school 2005, Revue Européenne de Génie Civil, vol. 9, n 5-6, 2005b, p. 651-670.

Laloui L., Cekerevac C., François B., "Constitutive modelling of the thermo-plastic behaviour of soils”, ALERT autumn school 2005, Revue Européenne de Génie Civil, vol. 9, n 5-6, 2005c, p. 635-650.

Laloui L., Cekerevac C., "Non-isothermal Plasticity Model for Cyclic Behaviour of Soils". International Journal of Numerical and Analytical Methods in Geomechanics, vol. 32, $\mathrm{n}^{\circ} 5$, 2008, p. 437-460.

Laloui L., François B., Nuth M., Péron H., Koliji, A. “A thermo-hydro-mechanical stressstrain framework for modelling the performance of clay barriers in deep geological repositories for radioactive waste“. Unsaturated Soils: Advances in Geo-Engineering, Toll et al. (eds), pp. 63-80, 2008.

Laloui L., François B., "ACMEG-T: A Soil Thermo-Plasticity Model", Journal of Engineering Mechanics, doi: 10.1061/(ASCE)EM.1943-7889.0000011, 2009.

Liu Z., Boukpeti N., Li X., Collin F., Radu J.P., Hueckel T., Charlier R., "Modelling chemohydro-mechanical behaviour of unsaturated clays: a feasibility study", International Journal for Numerical and Analytical Methods in Geomechanics, vol. 29, n 9, 2005, p. 919-940.

Masekanya J-P. Stabilté des pentes et saturation partielles: Etude expérimentale et modélisation numérique. Doctoral Thesis, University of Liège, 2008. 
Modaressi H., Laloui L., “Thermo-viscoplastic model for clays”, International Journal of Numerical and Analytical Methods in Geomechanics, vol. 21, 1997, p. 313-335.

Nova R., Parma M., “An instructive chemo-mechanical model for bonded geomaterials”, Revue Européenne de Génie Civil, 2005.

Nuth M., Laloui L. "Effective Stress Concept in Unsaturated Soils: Clarification and Validation of a Unified Framework”, International Journal of Numerical and Analytical Methods in Geomechanics, vol. 32, n, 2008, p. 771-801.

Priol G., De Gennaro V., Delage P., Cui Y.J., "On the suction and the time dependent behaviour of reservoir chalks of North sea oilfields", Proc. Second International Workshop on Unsaturated Soils, Capri (Italy), 2004, p. 43-54.

Schrefler B., The finite element method in soil consolidation (with application to surface subsidence). Doctoral thesis, CPH7684 University college of Swansea, 1984.

Terzaghi K., Theoretical soil mechanics, London, Chapman and Hall, 1943.

Turska E., Schrefler B.A., “ On convergence conditions of partitioned solution procedures for consolidation problems ”, Comp. Meth. InAppl. Mech. And Eng., vol. 106, 1993, p. 51-63.

Van Eekelen H.A.M., "Isotropic yield surfaces in three dimensions for use in soil mechanics”. International Journal for Numerical and Analytical Methods in Geomechanics, vol. 4, 1980, p. 98-101.

Vaunat J., Cante J.C., Ledesma A., Gens A., "A stress point algorithm for an elastoplastic model in unsaturated soils”, International Journal of Plasticity, vol. 16, 2000, p. 121-141.

Vulliet L., Laloui L., Schrefler B, Environmental geomechanics, EPFL-Press, 2002.

Zienkiewicz O.C., Paul D.K., Chan A.H.C., "Unconditionally stable staggered solution procedure for soil-pore fluid interaction problems”, Int. J. for Num. Meth. In Engineering, vol. 26, 1988, p. 1039-1055. 